\title{
Evaluación del daño renal por cadmio en población expuesta a contaminación por éste en agricultores de Quila-Quila, Potosí
}

\author{
Evaluation of kidney damage by cadmium in a population exposed to its contamination in farmers from \\ Quilla-Quilla, Potosí
}

\author{
C. Erostegui ${ }^{1}$, Carla Oporto², Lourdes Zalles ${ }^{3}$, Ricardo Sevilla ${ }^{1}$, Ana María Romero ${ }^{2}$
}

\begin{abstract}
Resumen
El cadmio es un metal pesado presente en desechos mineros y otros, y tiene efectos deletéreos en la salud humana especialmente en el riñón en el que destruye células tubulares. En una investigación de la calidad del lecho de los ríos que reciben aguas residuales en el norte de Potosí, Bolivia, se reportó presencia de cadmio en papas regadas con esas agua en Quila-quila, una población rural de la zona. Objetivo de este trabajo fue el de identificar posibles daños del cadmio en la salud de los pobladores de Quila-quila. Métodos: para ello se realizó revisión clínica y análisis laboratorial clínico y químico de sangre y orina de los pobladores de Quila-quila, y se calculó la tasa de filtración glomerular (TFG) y se correlacionó ésta con indicadores de lesión glomerular. Resultados: se encontraron niveles elevados de cadmio en sangre en algunas personas, y una correlación de la TFG con la concentración de cadmio en sangre y con indicadores de lesión de membrana glomerular. Conclusiones: en conclusión, los pobladores de Quila-quila estuvieron contaminados por cadmio, el cual produjo lesión renal leve.
\end{abstract}

Palabras claves: cadmio, contaminación ambiental, tasa de filtración glomerular

\section{Abstract}

Cadmium is a heavy metal present in mining and other wastes, and has deleterious effects on human health especially in the kidney in which it destroys tubular cells. In an investigation of the quality of the river beds that receive wastewater in the north of Potosí, Bolivia, the presence of cadmium was reported in potatoes watered with these water in Quila-quila, a rural town in the area. The objective of this work was to identify possible damage from cadmium to the health of the inhabitants of Quila-quila. Methods: for this, clinical review and clinical and chemical laboratory analysis of blood and urine from the inhabitants of Quila-quila, and the filtration rate was calculated glomerular (GFR) and this was correlated with indicators of glomerular injury. Results: elevated levels of cadmium were found in the blood in some people, and a correlation of GFR with blood cadmium concentration and indicators of membrane injury glomerular. Conclusion: in conclusion, the inhabitants of Quila-quila were contaminated by cadmium, which caused mild kidney damage.

Palabras claves: cadmium, environmental pollution, glomerular filtration rate

$\mathrm{E}^{\mathrm{l}}$ 1 cadmio es un metal pesado común en áreas mineras y en áreas industriales sin función biológica conocida. Es considerado como un contaminante ya que su acumulación produce daño biológico al inhibir los grupos sulfhidrilo $(\mathrm{SH})$ que intervienen en la mayoría de los procesos enzimáticos de nuestro organismo.

El cadmio puede ingresar al organismo a través de los pulmones, del sistema digestivo o de la piel. La mayor parte se deposita en el hígado y principalmente en los riñones en los que produce mayor daño al lesionar las células tubulares proximales por acumulación crónica ${ }^{1}$. Su exceso puede causar la muerte. Se elimina esencialmente por vía renal, intestinal y

${ }^{1}$ Instituto de Investigaciones Biomédicas, Facultad de Medicina, UMSS, Cochabamba Bolivia. https://orcid.org/0000-0002-5071-2992,

https://orcid.org/0000-0002-8236-4048.

${ }^{2}$ Centro de Aguas y Saneamiento Ambiental. Facultad de Ciencia y Tecnología, UMSS. Cochabamba, Bolivia.

${ }^{3}$ Laboratorios médicos "LABIMED", Facultad de Medicina, UMSS, Cochabamba, Bolivia. https://orcid.org/0000-0002-6593-2891.

${ }^{*}$ Correspondencia a: Carlos Erostegui

Correo electrónico: carl.erostegui@umss.edu

Recibido el 22 de agosto de 2020. Aceptado el 13 de octubre de 2020 por las faneras.

La intoxicación aguda por cadmio puede ser detectada mediante la medición directa de este metal en sangre y orina. La acumulación crónica está asociada con disfunción renal, y actualmente se acepta que el indicador más sensitivo y específico para la detección de falla renal por cadmio es la disminución de la reabsorción tubular de proteínas de bajo peso molecular1. Los marcadores más comunes de alteración renal son la creatinina sérica beta2-microblobulina, la proteína ligada al retinol y la alfa1-microglobulina ${ }^{2,3}$. Por otra parte, la tasa de filtración glomerular (TFG) es un parámetro importante, pues indica la capacidad de filtración de los riñones y por tanto, el estado de la membrana glomerular.

La TFG puede ser determinada con precisión a partir de la creatinina sérica mediante ecuaciones ${ }^{4,5}$. Recientemente se ha reportado la asociación de altos niveles de cadmio con niveles de creatinina sérica ${ }^{6}$, por lo que los posibles efectos del cadmio en el riñón pueden ser calculados mediante la obtención de valores de creatinina. Se estima que los niveles mínimos de Cd urinario varían de 2 a 10 microgramos/gramo de creatinina.

Quila-quila es un pueblo del altiplano boliviano situado en una zona de explotación minera en la que los ríos están 


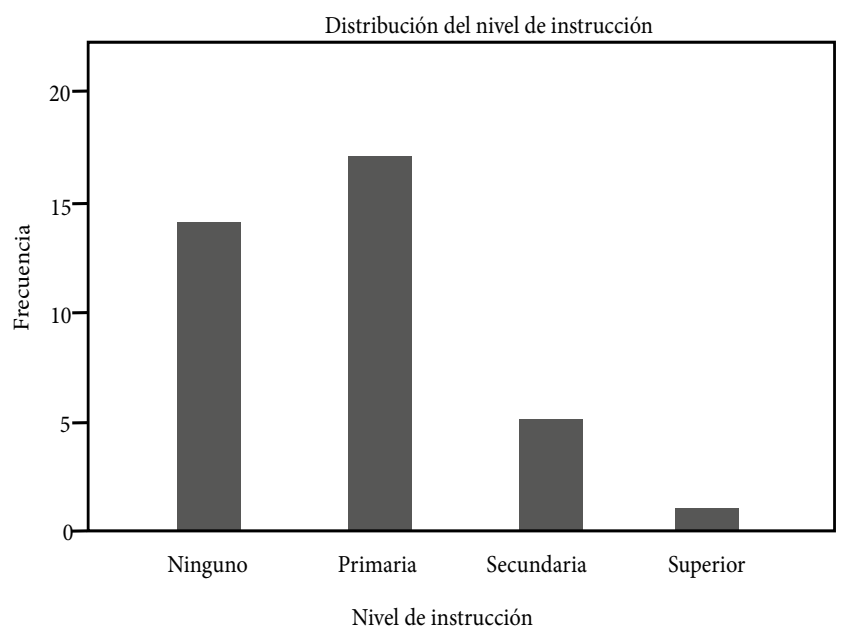

Figura 1. Grafica del nivel de instrucción de los pobladores de Quila-quila

contaminados con aguas que provienen de los desechos y lavados de las minas e ingenios metalúrgicos. Los habitantes de Quila-quila cultivan sus alimentos, principalmente papa, y haba con esta agua contaminada. En un estudio realizado por Oporto se ha encontrado en las papas y haba cultivada en la región, niveles de cadmio elevado y la ingesta de $\mathrm{Cd}$ calculada excedía el máximo recomendado por la $\mathrm{OMS}^{7}$, por lo que se pensó que los habitantes de Quila-quila estuvieran contaminados con cadmio y tuvieran una prevalencia alta de daño renal, ya que la producción de papa y haba es consumida por ellos mismos. El objetivo del trabajo fue el determinar la incidencia de patología renal causada por contaminación crónica por cadmio en la población de Quila-quila expuesta a éste.

\section{Material y métodos}

Tipo de estudio observacional, transversal, realizado en la población de Quila Quila-Potosi, con muestreo no probabilístico, accidental.

Para la evaluación de la patología renal y posible asociación con los niveles de cadmio en los pobladores de Quila quila, se procedió a la revisión médica y análisis de sangre y orina. La revisión clínica se realizó en el consultorio del centro de salud de la población de Quila-quila. Se tomaron los datos demográficos, medidas antropométricas, una anamnesis de la dieta, medición de los parámetros clínicos como PA, FC, temperatura, exploración física en busca de signos patológicos generales y específicos del sistema genitourinario, y neurológico.

La muestra de sangre fue tomada de sangre venosa y conservada a $4^{\circ} \mathrm{C}$ durante su transporte hasta el laboratorio, donde se realizó hemograma completo, perfil lipídico, perfil hepático y perfil renal, determinación de Beta-2microglobulina albúmina y ferritina. Se determinaron niveles plasmáticos de colesterol total, triglicéridos, HDL, LDL, creatinina, urea, ácido úrico y fosfatasa alcalina, transaminasa GOT, transaminasa GPT mediante análisis con Stat fax 3300.

Las pruebas de orina fueron, parcial de orina, y examen
Tabla 1. Características clínicas de la población de estudio

\begin{tabular}{lrc}
\hline & Media & \multicolumn{1}{c}{ DE } \\
\hline Edad (años) & 58,06 & 18,235 \\
Talla (cm) & 152,42 & 11,497 \\
\hline Peso (kg) & 53,58 & 8,911 \\
\hline Perímetro Cefálico. (cm) & 54,14 & 1,536 \\
\hline Perímetro Braquial (cm) & 24,09 & 2,930 \\
\hline Pliegue tricipital (cm) & 8,55 & 4,928 \\
\hline IMC & 23,4431 & 5,45984 \\
\hline Presión arterial sistólica (mmHg) & 107,361 & 8,55278 \\
\hline Presión arterial diastólica (mmHg) & 61,4722 & 17,5355 \\
\hline
\end{tabular}

químico microscópico, determinación de la Beta-2microglobulina, microalbumina y creatinina.

La concentración de cadmio en sangre y orina se determinó mediante espectrofotometría de absorción atómica, previa digestión de la materia orgánica de las muestras, en horno de microondas Milestone-Ethos 900. Las lecturas fueron realizadas en un Espectrómetro de Absorción Atómica Perkin Elmer AAnalyst 700 con sistema de atomización electrotérmica PU 9095, y como gas de purga el Argón.

Todas las técnicas laboratoriales fueron estandarizadas previamente con sueros controles y estándares o calibradores propios de los kits comerciales. El control de calidad de las pruebas de química sanguínea (enzimáticas y cinéticas) fue realizado con controles de nivel 1 y nivel 2 .

El análisis de la función renal se realizó en base a los parámetros directos medidos en sangre y orina, y se calculó la tasa de filtración glomerular mediante la ecuación de Levey en base a la creatinina sérica, edad, y otros parámetros ${ }^{4,5}$, cuya ecuación se muestra a continuación.

\section{GFR $=170^{\star}$ SCr $-0,999 *$ edad $-0,176^{\star}$ BUN $-170^{\star}$ album 0,318}

Donde GFR es la tasa de filtración glomerular; SCr, el nivel de creatinina sérica y BUN la concentración de nitrógeno de urea en sangre.

\section{Resultados}

La población de Quila-quila está compuesta en general por personas mayores y niños. La mayor parte de la población adulta joven ha emigrado principalmente en busca de trabajo y vive en ciudades más grandes como Llallagua y Oruro (Figura 1).

De la población estudiada, el nivel de instrucción de la mayor parte es bajo, (primaria o ninguno), lo cual corresponde con la edad de la población, pues anteriormente eran muy pocos los que acudían a la escuela. La mayor parte (67\%) son originarios de Quila-quila; El promedio de hijos por persona es de 5,6; La ocupación principal es la de agricultor, seguida 
A Relación de la concentración de Cd en sangre y orina en función de la edad

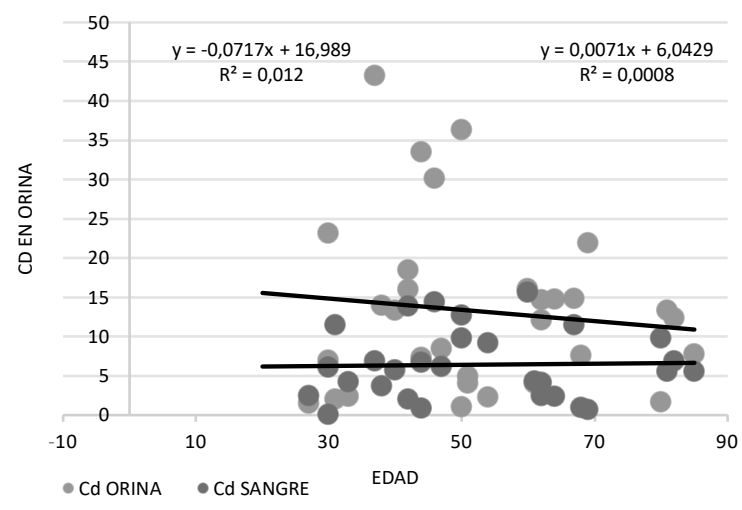

B

Relacion de la concentración de cd en orina vs. sangre

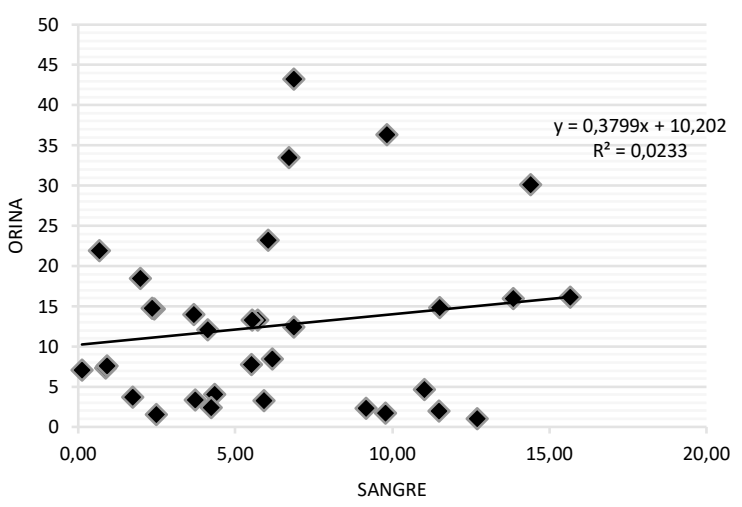

Figura 2. Gráfica que muestra la correlación de la edad con la concentración de cadmio en orina y sangre y entre la concentracion de cadmio en orina con la de sangre. (A) Aparentemente los niveles de cadmio son mayores en orina de personas entre 30 y 50 años, aunque los datos muestran gran dispersión, mientras que en sangre la diferencia es mínima. (B)Los que tienen altos niveles de cadmio en orina tienen niveles medios en sangre, y son muy pocos los que tiene altos niveles en ambos.

de ama de casa.

Los resultados promedio de la medición de los diferentes parámetros antropométricos y clínicos se muestran en la siguiente Tabla 1.

Estas mediciones (Tabla 1) nos muestran un panorama general del estado de salud de la población estudiada que podemos describirla como de edad madura, de baja talla, con peso bajo, pero con IMC aumentada, 6 personas tienen obesidad y cursan con hiperlipidemia. Es decir que, desde el punto de vista nutricional, es una población mal nutrida crónicamente con sobrepeso. En la anamnesis encontramos PA dentro de rangos normales, pero menor al promedio de la población general. Del examen del sistema cardiovascular muestra que no existen problemas a este nivel. El análisis del sistema nervioso y osteo-muscular muestra que 7 personas tenían síntomas de artritis reumatoide. La patología que se observa con mayor frecuencia desde el punto de vista clínico, entre los pobladores de Quila-quila, es la infección urinaria $(\mathrm{N}=11)$ y una posiblemente con nefropatía.

\section{Efecto del cadmio}

Los niveles de cadmio encontrados en los pobladores de Quila-quila fueron: 6,32 ( $\pm 4,33 \mathrm{SEM})$ en plasma y 12,14 ( $\pm 10,6 \mathrm{SEM})$, en orina, los niveles permitidos de Cd en sangre son hasta $10 \mathrm{ug} / \mathrm{L}$. Los niveles de cadmio se correlacionaron con diferentes parámetros de la población, para determinar si alguno de los factores estaba alterado por la posible acumulación del cadmio. No se encontró correlación entre la edad y la concentración de cadmio en sangre o en orina, tampoco se encuentra correlación entre los niveles de cadmio en sangre y orina (Figura 2).

La lesión de la membrana glomerular es expresada por la cantidad de proteína en orina, y específicamente la albúmina

\section{A}

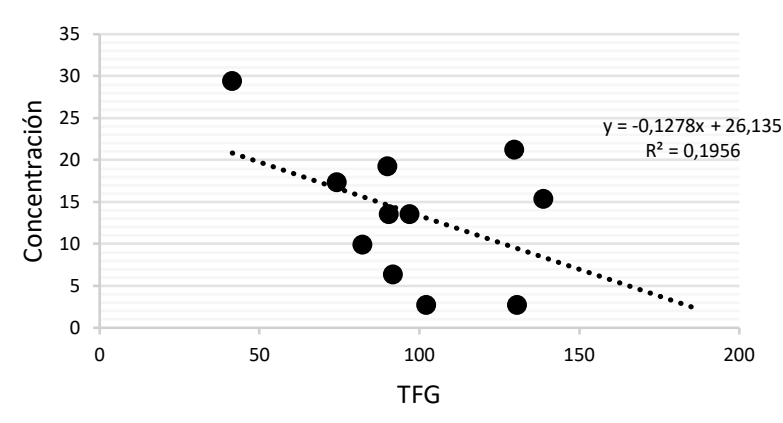

B

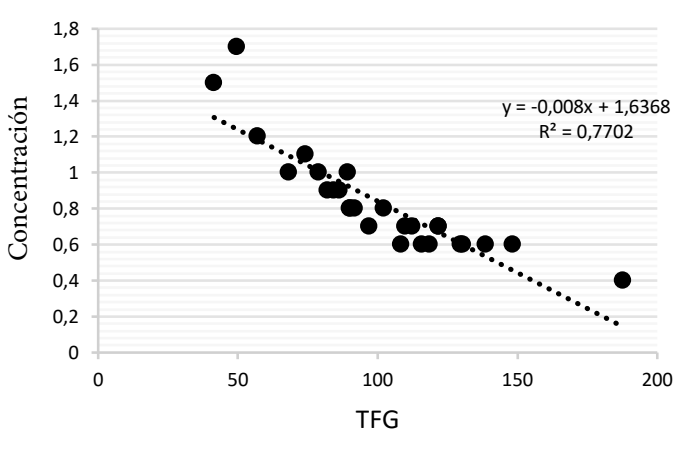

Figura 3. Correlacion entre la tasa de filtrado glomerular (TFG) calculado con la ecuación de Levey y la concentración de albúmina (A) y creatinina (B) en los pacientes de Quila- quila. La correlación para la creatinina mostrada en B es evidente con valores muy bajos de creatinina para una TFG alta, y correlación menos evidente pero positiva para la albúmina, lo que nos indica un daño glomerular en pacientes con TFG menor a $100 \mathrm{ml} / \mathrm{min}$. 
o microglobulinas, y la relación entre las conentraciones plasmáticas y en orina es un indicador de la extensión del daño glomerular. En nuestro estudio, ninguno de los pacientes tiene un estado clínico de insuficiencia renal, pero 3 de 34 pacientes tienen albumina aumentada (> a $20 \mathrm{ug} / \mathrm{ml}$ ).

La presencia de proteínas plasmáticas en orina, especialmente la albúmina, es un indicador de lesión glomerular, pues indica ruptura de la barrera glomerular (endotelio y capsula de Bowman). El valor de la relación de la concentración de albúmina plasmática sobre la de orina debe ser siempre mayor a 1 , un valor menor indica que la cantidad de albumina en orina es mayor que la plasmática, por tanto, ésta está siendo filtrada y se pierde por la orina debido a lesión de la membrana glomerular. En nuestro estudio, la relación entre estos dos parámetros muestra que en 12 de 36 (33\%) pacientes fue menor a 1, es decir que un tercio de los pacientes tiene lesión de la membrana glomerular. Es sabido que la acumulación del cadmio en el riñon produce lesión membranal, y por tanto afecta el índice de filtración glomerular. En los pacientes estudiados, se calculó el índice de filtración glomerular (TFG) con la formula de Levey ${ }^{5}$ y se calculó la correlación TFG y concentración de albúmina, rfelación validada por la correlacion alta entre TFG y concentraciòn de creatinina (figura 3), encontrandose una correlación con $\mathrm{R}^{2}$ de 0,19 entre la presencia de albúmina y la TFG, indicando tambien que en los pacientes con presencia de albumina alta en orina, la TFG está disminuida, por tanto la membrana glomerular dañada.

Por otra parte, la microglobulina es un indicador de actividad de células del sistema inmune, pero también de la reabsorción tubular ${ }^{8,9}$. En nuestro estudio, 19 de 34 pacientes $(55,8 \%)$, tenían microglobulina aumentada en la orina.

\section{Discusión}

Nuestros datos muestran que la población de Quila- quila tiene condiciones socioeconómicas deficientes, principalmente en cuanto a la educación. Se esperaba, partiendo de la hipótesis de que los productos alimenticios producidos en la zona, regados con agua contaminada con cadmio proveniente del rio el cual contiene aguas de las minas cercanas, y consumidos por la población de Quila-quila, tendrían cadmio acumulado ${ }^{7}$ y posiblemente afectando a la función renal. Esto se confirma en un tercio de la población estudiada, en la que se encontró niveles altos de cadmio en orina. Sin embargo, por las características productivas de la zona, parte del año (en la época seca), la población reside en ciudades cercanas, y por tanto no consume los alimentos producido en la zona y así se detienen el proceso de contaminación, razón por la que no encontramos niveles elevados que indiquen exposición aguda.

Por otra parte, sabemos que el cadmio se acumula en el riñón y altera el filtrado glomerular. En el estudio no se realizó biopsia renal, pero si pudimos ver que en la población estudiada hay un porcentaje alto que tienen filtrado glomerular disminuido, (calculada con la ecuación de Levey), aún en personas jóvenes. Esto nos lleva a pensar que efectivamente un cierto porcentaje de la población tiene lesión renal. Nuestros cálculos basados en la ecuación de Levey ${ }^{5}$, parecen confiables, pues son validados por la buena correlación entre la TFG con los niveles de creatinina. Los niveles de albumina también indican lesión de la membrana glomerular en cierto porcentaje de la población. La baja correlación que encontramos respecto a la albúmina, y a la alfa y beta microglobulina, es real, e indica que efectivamente el cadmio afecta más a la membrana glomerular que a la función tubular.

Conflicto de intereses: los autores declaran que no existe conflicto de intereses.

\section{Referencias bibliográficas}

1. Bernard, A. (Oct de 2004). Renal dysfunction induced by cadmium: biomarkers of critical effects. Biometals., 17(5), 19-23.

2. Roels, H., Hoet, P., \& Lison, D. (Mayo-Julio de 1999 ). Usefulness of biomarkers of exposure to inorganic mercury, lead, or cadmium in controlling occupational and environmental risks of nephrotoxicity. Ren Fail.., 21((3-4)), 251-62Chakraborty, S., Dutta, A. R., Sural, S., \& Sen, D. G. ( 2013 ). Ailing bones and failing kidneys: a case of chronic. Ann Clin Biochem Online, 10.1177/0004563213481207.

3. Järup, L., Berglund, M., Elinder, C., Nordberg, G., \& Vahter, M. (1998). Health effects of cadmium exposure--a review of the literature and a risk estimate. Scand J Work Environ Health., 24 Suppl 1:1-51.
4. Earley Amy, M. D. (2012). Estimating equations for glomerular filtration rate in the era os creatinine standarization. Ann Intern Med(156), 785-795.

5. Earley, Miskulin, Lamb, Levey, \& Uhlig. (2012). Estimating equations for glomerular filtration rate in the era of creatinine standarization. Annals of Internal Medicina, 785-795.

6. Weaver Virginia, K. N.-S.-K. (november de 2011 ). Differences in urine cadmium associations with kidney outcomes based on serumcreatinine and cystatin c. (Elsevier, Ed.) Environ Res., 11(8), 1236-1242.

7. Oporto, C., Vandecasteele, C., \& Smolders., a. E. ( JULY-AUGUST de 2007). Elevated Cadmium Concentrations in Potato Tubers Due to Irrigation with River Water. J. ENVIRON. QUAL, VOL. 36,.
8. Donadio, C. (2010). Serum and urinary markers of early impairment of GFR in chronic kidney. Am J Physiol Renal Physiol 299:, F1407-F1423.

9. Zeng, X., Hossain, D., Bostwick, D. G., Herrera, G. A., \& Zhang4, a. P. (2014). Urinary $\bigotimes 2$ Microglobulin Is a Good Indicator of Proximal. Journal of Biomarkers. Volume 2014, http:// dx.doi.org/10.1155/2014/492838.

10. Chakraborty, S., Dutta, A. R., Sural, S., Gupta, D., \& Sen, a. S. (2013). Ailing bones and failing kidneys: a case of chronic. Ann Clin Biochem Online.

11. Ramírez, A. V. (2006; 67(1)). Biomarcadores en monitoreo de exposición a metales. An Fac Med Lima , 48-58. 